\title{
Prognostic value of heterogeneous ribonucleoprotein A1 expression and inflammatory indicators for patients with surgically resected hepatocellular carcinoma: Perspectives from a high occurrence area of hepatocellular carcinoma in China
}

\author{
RUISHENG KE ${ }^{1 *}$, LIZHI LV ${ }^{1,2^{*}}$, JIAYAN LI ${ }^{3}$, XIAOJIN ZHANG ${ }^{2}$, \\ FANG YANG $^{2}$, KUN ZHANG ${ }^{1,2}$ and YI JIANG ${ }^{1,2}$ \\ ${ }^{1}$ Department of Hepatobiliary Surgery, The Fuzong Clinical Medical College of Fujian Medical \\ University; ${ }^{2}$ Department of Hepatobiliary Surgery, Fuzhou General Hospital; ${ }^{3}$ Department of Surgery, \\ Fuzhou Hospital of Traditional Chinese Medicine, Fuzhou, Fujian 350025, P.R. China
}

Received December 3, 2017; Accepted May 16, 2018

DOI: $10.3892 / \mathrm{ol} .2018 .9079$

\begin{abstract}
Heterogeneous ribonucleoprotein A1 (hnRNPA1) is a documented tumor biomarker known to be aberrantly expressed in a number of types of human cancer. However, to the best of our knowledge, its prognostic value for surgically resected HCC (RHCC) in the high incidence areas of China has not been described; the association between hnRNPA1 expression, pre-operative neutrophil to lymphocyte ratio (NLR) and platelet to lymphocyte ratio (PLR) is also not understood. In the present study, hnRNPA1 expression was retrospectively measured in two independent cohorts of patients with hepatocellular carcinoma (HCC) who underwent surgery to remove the primary cancer at one center in Fujian, an area with a high incidence of HCC in China. Reverse transcription-quantitative polymerase chain reaction (RT-qPCR), western blotting and immunohistochemistry (IHC) were used to quantify hnRNPA1 expression in RHCC tissues. The survival curves were plotted using the Kaplan-Meier method, and the prognostic significance of hnRNPA1, NLR and PLR was analyzed using the log-rank test. The relevant prognostic factors
\end{abstract}

Correspondence to: Professor Kun Zhang or Professor Yi Jiang, Department of Hepatobiliary Surgery, Fuzhou General Hospital, 156 North Xi-Er Huan Road, Fuzhou, Fujian 350025, P.R. China E-mail: zhangkun73@aliyun.com

E-mail: jiangyi8183@163.com

*Contributed equally

Key words: heterogeneous ribonucleoprotein A1, hepatocellular carcinoma, neutrophil to lymphocyte ratio, platelet to lymphocyte ratio, prognosis were identified by multivariate Cox regression analysis. RT-qPCR and western blotting revealed that hnRNPA1 was upregulated in $\mathrm{HCC}$ tissues $(\mathrm{P}<0.001)$, and particularly overexpressed in tumor tissues of patients with recurrent HCC $(\mathrm{P}<0.001)$ (cohort 1; 54 patients). Differential hnRNPA1 expression was measured in 426HCC tissues with IHC; 259 exhibited high hnRNPA1 expression and 167 exhibited low expression. High hnRNPA1 expression was significantly associated with Tumor-Node-Metastasis stage $(\mathrm{P}=0.024)$, tumor size $(\mathrm{P}=0.027)$, vascular invasion $(\mathrm{P}<0.001)$, Edmonson grade $(\mathrm{P}<0.001)$, pre-operative serum $\alpha$-fetoprotein $($ AFP) $(\mathrm{P}<0.001)$, NLR $(\mathrm{P}<0.001)$ and PLR $(\mathrm{P}<0.001)$. In addition, multivariate Cox regression analysis confirmed that high hnRNPA1 expression was associated with relapse-free survival (RFS; HR, 0.685; 95\% CI, 0.506-0.928; $\mathrm{P}=0.015$ ) and overall survival (OS; HR, 0.629; 95\% CI, 0.454-0.871; $\mathrm{P}=0.005)$. Multivariate analysis confirmed that higher pre-operative serum AFP had an unfavorable impact on RFS (HR, 1.350; 95\% CI, 1.006-1.811; $\mathrm{P}=0.045)$ and $\mathrm{OS}(\mathrm{HR}=1.564 ; 95 \% \mathrm{CI}$, 1.151-2.126; $\mathrm{P}=0.004)$, while higher pre-operative NLR had an unfavorable impact on OS (HR, 1.758; 95\% CI, 1.161-2.661; $\mathrm{P}=0.008$ ) (cohort 2;426 patients). The expression of hnRNPA1 was also positively correlated with NLR (Spearman's correlation; $r=0.122, P=0.012$ ) and PLR (Spearman's correlation; $r=0.140, \mathrm{P}=0.004)$. In conclusion, high hnRNPA1 expression was revealed as prognostic for poor survival in patients with RHCC, and detection of hnRNPA1 protein in tumor tissues demonstrated potential in estimating survival for patients with RHCC in areas with high incidence rates. Furthermore, the combination of high hnRNPA1 expression and pre-operative serum AFP levels $(>400 \mu \mathrm{g})$ proved to be a good diagnostic and prognostic biomarker for this specific population of patients. Finally, a correlation may also exist between hnRNPA1 expression and other markers of systemic inflammation. 


\section{Introduction}

HCC causes $\sim 1$ million mortalities worldwide annually and half of these mortalities occur in China (1). The areas of high HCC occurrence in China are concentrated in the southeast coastal areas, including Fujian (2). The complicated signal transduction pathways and underlying molecular pathogenesis involved in HCC are not fully understood. Several molecular markers have been proposed that may identify the metastatic potential and risk of recurrence of HCC; however, none have been approved for routine clinical use (3). Although pre-operative serum $\alpha$-fetoprotein (AFP) is a commonly used diagnostic and prognostic marker for $\mathrm{HCC}$, not all patients exhibit elevated AFP levels at the pre-operative stage (4). Therefore, other biomarkers or similar indicators are required that can be combined with AFP for prognostic and diagnostic use in HCC. Biological indicators associated with the recurrence of liver cancer subsequent to surgery are particularly significant in clinical practice (5). A number of tumor suppressors and oncogenes have been demonstrated to promote the transformed phenotype of cancer cells (6), along with splicing factors that have been previously confirmed as important participants in cancer development (7). Heterogeneous ribonucleoproteins (hnRNPs) area nuclear protein class that bind to nascent RNA transcripts, with a variety of roles in telomere biogenesis, DNA repair, cell signaling and the regulation of gene expression at the transcriptional and translational levels (8). hnRNPA1 belongs to a family of hnRNPs that function as splicing factors, and is involved in telomere biogenesis; telomeres of an hnRNPA1-deficient cell line were previously revealed to be shorter than those of a associated hnRNPA1-expressing cell line (9). In recent years, hnRNPA1 overexpression has been observed in various malignancies, and has been identified as a potential biomarker for early diagnosis, prognosis and monitoring during treatment of colorectal cancer (10-13). However, a previous study reported its prognostic value for surgically resected HCC (RHCC) (14), particularly in areas of high HCC occurrence in China. Studies have also demonstrated lymphatic infiltration caused by chronic inflammatory responses in the tumor and its surrounding tissues as a possible underlying cause of tumorigenesis (15); since the neutrophil to lymphocyte ratio (NLR) and the platelet to lymphocyte ratio (PLR) are the markers of immune status, they also reflect the inflammatory state and are associated with survival prognosis $(16,17)$. The correlation between inflammation indicators and hnRNPA1 in HCC is not yet clear. To address this, the present study investigated the significance of hnRNPA1 in two cohorts of patients with RHCC in Fujian, where HCC is common the clinicopathological data of all patients were collected, and hnRNPA1 expression was determined with reverse transcription-quantitative polymerase chain reaction (RT-qPCR), western blotting and immunohistochemistry (IHC). The correlations between hnRNPA1 expression and pre-operative serum AFP levels, NLR and PLR were also determined.

\section{Materials and methods}

Patients and follow-up. Two independent cohorts of patients with HCC were enrolled for the study. This due to the different specimens we collected. Between June 2014 and February 2015, fresh liver cancer tissue samples were collected, which were used for RT-qPCR and western blot analyses. However, prior to March 2012, only collect paraffin-embedded liver cancer specimens, that were used for IHC were available. To determine the expression of hnRNPA1 in HCC tissues, 54 cancerous and pericancerous liver tissues (PCLT) were collected for RT-qPCR and western blot analyses (cohort 1). The samples were collected from patients with HCC who underwent curative resection at the Department of Hepatobiliary Surgery, Fuzhou General Hospital (Fujian, China), between June 2014 and February 2015. These patients were monitored following surgery until August 15, 2017. All specimens were collected immediately after resection, transported in liquid nitrogen and stored at $-80^{\circ} \mathrm{C}$. To evaluate the prognostic role of hnRNPA1 in HCC, tumor specimens for IHC analyses were obtained from 426 consecutive patients with HCC who underwent curative resection between January 2002 and March 2012 (cohort 2). These patients were evaluated from the day of surgical resection to the last day of follow-up (August 15, 2017), with a median follow-up time of 59 months (range, 2.0-128.0 months). Liver function and tumor differentiation were assessed by Child-Pugh classification (18) and the Edmondson grading system (18), respectively. Tumor stages were assessed according to the 2010 International Union Against Cancer Tumor-Node-Metastasis (TNM) classification system (19), and curative resection was defined as previously described (14). Overall survival (OS) was defined as the interval between surgery and mortality or between surgery and the last observation point. For surviving patients, the data were censored at the last follow-up. Relapse-free survival (RFS) was defined as the interval between the date of surgery and the date of diagnosis of any type of relapse (intra-hepatic recurrence or extrahepatic metastasis). The inclusion criteria included the following: ECOG score (18) of 0-1 prior to surgery, absence of ascites, hepatic encephalopathy and any distant metastasis, only one tumor lesion and Child-Pugh class A. Clinicopathological data of the patients with HCC (Table I) were collected from the hospital database medical records. Survival information was obtained from medical records, telephone interviews and the Social Security Death Index (18), and all pathological data were pathological characteristics originally assessed by pathologists.

$R T-q P C R$. Total RNA was extracted from HCC tissues using TRIzol Reagent (Invitrogen; Thermo Fisher Scientific, Inc., Waltham, MA, USA) according to the manufacturer's protocols. cDNA was synthesized by HiScript reverse transcription kit (Vazyme Biotech Co., Ltd, Nanjing, China) and Real-time PCR SYBR Green kit (Vazyme Biotech Co., Ltd.) was used to prepare the protein according to the manufacturer's protocol. The negative control was used to replace the template with double distilled water and $\beta$-actin as control. The gene expression of hnRNPA1 and $\beta$-actin in from patient tissues was measured using One-Step RT-PCR Master mix (Thermo Fisher Scientific, Inc.). The primers used were: hnRNPA1 forward, 5'-TTTGACGACCATGACTCCGT-3' and reverse, 5'-CACGACCACCACCAAAGTTT-3; and $\beta$-actin forward, 5'-AGCGAGCATCCCCCAAAGTT-3' and reverse, 5'-GGG CACGAAGGCTCATCATT-3'. The reaction conditions were 
Table I. Clinicopathological characteristics of patients with hepatocellular carcinoma in two cohorts.

\begin{tabular}{|c|c|c|c|c|}
\hline \multirow[b]{2}{*}{ Characteristics } & \multicolumn{2}{|c|}{$\begin{array}{c}\text { Cohort } 1 \\
(\mathrm{n}=54)\end{array}$} & \multicolumn{2}{|c|}{$\begin{array}{l}\text { Cohort } 2 \\
(n=426)\end{array}$} \\
\hline & $\mathrm{n}$ & $\%$ & $\mathrm{n}$ & $\%$ \\
\hline \multicolumn{5}{|l|}{ Age, years } \\
\hline$\leq 55$ & 29 & 53.7 & 174 & 40.8 \\
\hline$>55$ & 25 & 46.3 & 252 & 59.2 \\
\hline \multicolumn{5}{|l|}{ Sex } \\
\hline Male & 45 & 83.3 & 378 & 88.7 \\
\hline Female & 9 & 16.7 & 48 & 11.3 \\
\hline \multicolumn{5}{|l|}{ Tumor location } \\
\hline Left & 15 & 27.8 & 101 & 23.7 \\
\hline Right & 39 & 72.2 & 325 & 76.3 \\
\hline \multicolumn{5}{|l|}{ TNM stage } \\
\hline $\mathrm{I} / \mathrm{II}$ & 37 & 68.5 & 292 & 68.5 \\
\hline IIIa & 17 & 31.5 & 134 & 31.5 \\
\hline \multicolumn{5}{|l|}{ Tumor size, cm } \\
\hline$\leq 5$ & 28 & 51.9 & 219 & 51.4 \\
\hline$>5$ & 26 & 48.1 & 207 & 48.6 \\
\hline \multicolumn{5}{|c|}{ Vascular invasion } \\
\hline Yes & 29 & 53.7 & 287 & 67.4 \\
\hline No & 25 & 46.3 & 139 & 32.6 \\
\hline \multicolumn{5}{|c|}{ Edmonson grade } \\
\hline I & 43 & 79.6 & 343 & 80.5 \\
\hline II-IV & 11 & 20.4 & 83 & 19.5 \\
\hline \multicolumn{5}{|l|}{$\mathrm{AFP}, \mu \mathrm{g} / \mathrm{l}$} \\
\hline$\leq 400$ & 32 & 59.3 & 267 & 62.7 \\
\hline$>400$ & 22 & 40.7 & 159 & 37.3 \\
\hline \multicolumn{5}{|l|}{ HBsAg } \\
\hline Negative & 35 & 64.8 & 275 & 64.6 \\
\hline Positive & 19 & 35.2 & 151 & 35.4 \\
\hline \multicolumn{5}{|c|}{ HBV DNA load, IU/ml } \\
\hline$\leq 104$ & 30 & 55.6 & 224 & 52.6 \\
\hline$>104$ & 24 & 44.4 & 202 & 47.4 \\
\hline \multicolumn{5}{|l|}{ NLR } \\
\hline$\leq 5.0$ & 18 & 33.3 & 125 & 29.3 \\
\hline$>5.0$ & 36 & 66.7 & 301 & 70.7 \\
\hline \multicolumn{5}{|l|}{ PLR } \\
\hline$\leq 91$ & 33 & 61.1 & 281 & 66.0 \\
\hline$>91$ & 21 & 38.9 & 145 & 34.0 \\
\hline \multicolumn{5}{|l|}{ Survival } \\
\hline Yes & NA & NA & 236 & 55.4 \\
\hline No & NA & NA & 190 & 44.6 \\
\hline \multicolumn{5}{|l|}{ Recurrence } \\
\hline Yes & 32 & 59.3 & 205 & 48.1 \\
\hline No & 22 & 40.7 & 221 & 51.9 \\
\hline
\end{tabular}

TNM, Tumor-Node-Metastasis; AFP, $\alpha$-fetoprotein; NLR, neutrophil to lymphocyte ratio; PLR, platelet to lymphocyte ratio; NA, not adopted; HBV, hepatitis B virus; HBsAg, HBV surface antigen; hnRNPA1, heterogeneous nuclear ribonucleoprotein A1. set to pre-denaturation at $95^{\circ} \mathrm{C}$ for $30 \mathrm{sec}, \mathrm{PCR}$ reaction at $94^{\circ} \mathrm{C}$ for $4 \mathrm{~min}, 94^{\circ} \mathrm{C}$ for $30 \mathrm{sec}, 56^{\circ} \mathrm{C}$ for $30 \mathrm{sec}, 72^{\circ} \mathrm{C}$ for $25 \mathrm{sec}$, dissolution curve conditions: $50^{\circ} \mathrm{C} 2 \mathrm{~min}, 95^{\circ} \mathrm{C} 10 \mathrm{~min} ; 95^{\circ} \mathrm{C}$ $30 \mathrm{sec}, 60^{\circ} \mathrm{C} 30 \mathrm{sec}, 40$ cycles. The expression of hnRNPA1 was measured relative to that of the $\beta$-actin standard and expressed as the $2^{-\Delta \Delta C \mathrm{q}}$ value (20).

Western blotting. The newly resected HCC and PCLT tissues were weighed and protein was extracted by protein lysis buffer (RIPA; Beyotime Institute of Biotechnology Co., Ltd. Shanghai, China). The protein concentrations were measured by the bicinchoninic acid method and $40 \mu \mathrm{g}$ protein was loaded into each lane on a $12 \%$ SDS-PAGE gel. Following electrophoresis, the protein bands were transferred to polyvinylidene fluoride (PVDF) membranes and blocked for $2 \mathrm{~h}$ at room temperature with PBS- $0.05 \%$ Tween 20 containing 5\% nonfat dry milk. The membranes were then incubated with the primary antibodies, rabbit anti-hnRNPA1 (ab177152; 1:4,000; Abcam, Cambridge, UK) and mouse anti- $\beta$-actin (BM0627; 1:200; Wuhan Boster Biological Technology, Ltd., Wuhan, China), overnight at $4^{\circ} \mathrm{C}$. The membranes were washed with Tris-buffered saline with Tween-20 (1X PBS, 0.1\% Tween-20) 3 times, and then incubated in HRP-conjugated goat anti-mouse (BA1051; 1:50,000; Wuhan Boster Biological Technology, Ltd, Wuhan, China) or goat anti-rabbit (BA1054; 1:50,000; Wuhan Boster Biological Engineering Co., Ltd, Wuhan, China) secondary antibodies in $1 \%$ nonfat dry milk in TBST for $1 \mathrm{~h}$ at room temperature. Membranes were washed 3 times in TBST and bound antibodies were detected using SuperSignal Chemiluminescent substrates (Thermo Fisher Scientific, Inc.) and chemiluminescence was detected on CL-XPosure Film (Thermo Fisher Scientific, Inc.). These data were analyzed via densitometry using Image-Pro plus v6.0 software (National Institutes of Health, Bethesda, MD, USA) and normalized to the expression of the internal control ( $\beta$-actin).

IHC. IHC methods published by Liu et al for a Her2 assay (21) were used to quantify hnRNPAlexpression in $426 \mathrm{HCC}$ tissue samples, with one modification: Anti-hnRNPA1 antibody (ab177152, 1:100; Abcam) replaced anti-HER-2 antibody. Pathological specimens from 426 HCC tissue samples were fixed in $4 \%$ neutral formaldehyde solution at room temperature for $24 \mathrm{~h}$, embedded in paraffin and sliced into 4-mm sections. Paraffin was removed with xylene (15 min; Sinopharm Group Chemical Reagent Co., Ltd., Shanghai, China) and the sections were rehydrated through a graded alcohol series (anhydrous ethanol I, $5 \mathrm{~min}$; anhydrous ethanol II, $5 \mathrm{~min}$; 95\% ethanol, $3 \mathrm{~min}$; 90\% ethanol, $3 \mathrm{~min}$; 80\% ethanol, $2 \mathrm{~min}$; and 70\% ethanol, 2 min) (Sinopharm Group Chemical Reagent Co., Ltd.). Antigen retrieval was performed by the microwave method. The sections were placed in a glass box filled with $10 \mathrm{mM}$ citrate buffer (pH 6.0; Shanghai Yanjing Reagent Technology Co., Ltd., Shanghai, China), and the glass box was placed in a microwave oven (700 W; Midea KJ23B-AN; Midea Group Co., Foshan, China) and heated at $100^{\circ} \mathrm{C}$ for $10-15 \mathrm{~min}$. After the repair solution is cooled to room temperature, the sections were removed and washed 3 times with PBS (pH 7.4) for $3 \mathrm{~min}$ each time. The sections were incubated overnight with the primary antibody anti-hnRNPA1 or TBS as negative control at $4^{\circ} \mathrm{C}$ for $30 \mathrm{~min}$. This was followed by incubation 
with a horseradish peroxidase (HRP)-conjugated secondary antibody (KIT-5010; anti-rabbit/mouse IgG; 1:50,000; Fuzhou Maixin Biotech Co., Ltd., Fuzhou, China) at $37^{\circ} \mathrm{C}$ for $30 \mathrm{~min}$. Chromagen detection was performed with 3,3'-diaminobenzidine and a substrate-chromogen (EnVision system; DAKO; Agilent Technologies, Inc., Santa Clara, CA, USA), followed by counter-staining with hematoxylin at room temperature for $1 \mathrm{~min}$. In every section, five randomly selected visual fields of view were assessed by light microscopy at x200 magnification (Olympus Corporation, Tokyo, Japan) to evaluate the expression on the expression of hnRNPA1. Positively stained cells were evaluated by two pathologists blinded to the patient data. Positive hnRNPA1 expression was localized to the nucleus of RHCC cells and was graded based on the number of stained cells. When $<10 \%$ cells were stained, the assessment was negative (-), while $\geq 10 \%$ staining was graded as positive (+). Among the positive samples, those with $10-25 \%$ positively stained cells were graded as + , those with $26-50 \%$ positive cells were graded as ++ and those with $51-75 \%$ positive cells were graded as +++ . A grade of ++++ indicted $>75 \%$ positively stained cancer cells. RHCC tissue samples with -, + or ++ grading were classified as low hnRNPA1 expression, whereas those with +++ and ++++ grading were classified as high hnRNPA1 expression.

Routine blood test. Blood samples were collected into EDTA tubes prior to surgical resection and routine blood tests were conducted to measure the pre-operative cell counts (total leukocytes, neutrophils, lymphocytes, platelets and erythrocytes). Hemoglobin counts were obtained from the patients medical records. Pre-operative NLR (22) and PLR (23) were measured, and their median values were found to be 5 and 91, respectively. The PLR and NLR values were used as a grouping threshold (high and low).

Statistical analysis. All statistical analyses were performed using SPSS statistical software version 21.0 (IBM Corp., Armonk, NY, USA) and Prism 5.0 (GraphPad Software, Inc., La Jolla, CA, USA). Student's unpaired t-test was used to compare the continuous variables data between groups. Associations between hnRNPA1 expression and patient clinicopathological characteristics were evaluated with the $\chi^{2}$ test. The correlation between hnRNPA1 expression and NLR/PLR was analyzed using Spearman's rank correlation test. The survival curves were plotted using Kaplan-Meier method and the prognostic significance of hnRNPA1 was analyzed using the log-rank test. Associations between hnRNPA1 expression, clinicopathological data and mortality risk were assessed using univariate regression analysis, and Cox multivariate regression analysis was used to determine independent prognostic factors. All statistical analyses were two-sided. $\mathrm{P}<0.05$ was considered to indicate a statistically significant difference.

\section{Results}

hnRNPAl is upregulated in HCC tissues. hnRNPA1 mRNA levels were measured in 54 paired HCC samples from cohort 1 by RT-qPCR. hnRNPA1 was revealed to be significantly overexpressed in tumor tissues compared with that in corresponding PCLT samples $(\mathrm{P}<0.001$; Fig. 1A). Samples from patients with tumor recurrences $(32 / 54)$ also revealed significantly higher levels of hnRNPA1mRNA than those from patients without recurrences (22/54; $\mathrm{P}<0.001$; Fig. 1B). The expression of hnRNPA1in 54 HCC cases from cohort 1wasalso assessed at the protein level by western blotting, and significant overexpression of hnRNPA1 protein was confirmed in tumors compared with that in corresponding PCLTs $(\mathrm{P}<0.001$; Fig. $1 \mathrm{C}$ and $\mathrm{D})$.

Association between patient clinicopathological features and hnRNPA1 expression. Patient clinicopathological data (cohort 1 and 2; Table I) and the associations with differential hnRNPA1 expression (cohort 2; Table II) were assessed. Positive hnRNPA1 expression was confirmed in cancer cell nuclei with IHC in both high and low hnRNPA1 expression groups in cohort 2 (Fig. 1E and F). High hnRNPA1 expression was significantly associated with elevated pre-operative blood AFP $(\mathrm{P}<0.001)$, TNM stage $(\mathrm{P}=0.024)$, tumor size $(>5 \mathrm{~cm})$ $(\mathrm{P}<0.001)$, vascular invasion $(\mathrm{P}<0.001)$, Edmonson grade $(\mathrm{P}<0.001)$, NLR $(\mathrm{P}=0.005), \mathrm{PLR}(\mathrm{P}=0.002)$, survival $(\mathrm{P}<0.001)$ and recurrence $(\mathrm{P}<0.001)$. Age, sex, tumor location, hepatitis $\mathrm{B}$ virus (HBV) surface antigen and HBV DNA load (>104 IU/ml) were not associated with hnRNPA1 expression (Table II).

Analysis of hnRNPAl expression during the observation period and RFS. Elevated hnRNPA1 expression $(\mathrm{P}<0.001)$, age $(\mathrm{P}=0.006)$, TNM stage $(\mathrm{P}<0.001)$, larger tumor size $(\mathrm{P}<0.001)$, vascular invasion $(\mathrm{P}<0.001)$, Edmonson grade $(\mathrm{P}=0.003)$, higher pre-operative serum AFP $(\mathrm{P}<0.001)$ and advanced NLR $(\mathrm{P}=0.031)$ were associated with recurrence in patients with $\mathrm{RHCC}$ during the observation period, as determined by Kaplan-Meier survival and log-rank analyses (Table III). Elevated hnRNPA1 expression was also significantly associated with shorter RFS during the same observation period; Fig. 2A demonstrates the different RFS times (in months) in the high and low hnRNPA1 expression groups $(\mathrm{P}<0.001)$. Univariate analyses confirmed that age (HR, 1.476; 95\% CI, 1.107-1.968; $\mathrm{P}=0.008)$, TNM stage (HR, 1.978; 95\% CI, 1.491-2.624; $\mathrm{P}<0.001)$, tumor size (HR, 1.898; 95\% CI, 1.438-2.506; $\mathrm{P}<0.001$ ), vascular invasion (HR, 6.850; 95\% CI, 4.352-10.780; $\mathrm{P}<0.001)$, Edmonson grade (HR, 0.563; 95\% CI, 0.381-0.831; P=0.004), pre-operative blood AFP $(\mathrm{HR}$, 1.742; 95\% CI, 1.320-2.300; P=0.001), NLR (HR, 1.394; 95\% CI, 1.023-1.899; $\mathrm{P}=0.035)$ and hnRNPA1 expression (HR, 2.295; 95\% CI, 1.688-3.120; $\mathrm{P}<0.001)$ were associated with RFS (Table IV). Multivariate analysis confirmed that age (HR, 0.654; 95\% CI, 0.488-0.877; $\mathrm{P}=0.005$ ), vascular invasion (HR, 6.125; 95\% CI, 3.835-9.870; $\mathrm{P}<0.001)$ and hnRNPA1 expression (HR, 0.685; 95\% CI, 0.506-0.928; $\mathrm{P}=0.015$ ) were independent prognostic factors of RFS (Table IV).

Analysis of hnRNPAl expression and OS during the observation period. Elevated hnRNPA1 expression $(\mathrm{P}<0.001)$, Edmonson grade $(\mathrm{P}<0.001)$, advanced TNM stage $(\mathrm{P}<0.001)$, tumor size $>5 \mathrm{~cm}(\mathrm{P}<0.001)$, vascular invasion $(\mathrm{P}<0.001)$, NLR $(\mathrm{P}<0.001), \mathrm{PLR}(\mathrm{P}<0.001)$ and higher pre-operative serum AFP $(\mathrm{P}<0.001)$ were associated with worse OS for patients with RHCC (Table III). Univariate analysis confirmed that TNM stage (HR, 2.357; 95\% CI, 1.770-3.140; P<0.001), tumor size (HR, 2.270; 95\% CI, 1.690-3.050; $\mathrm{P}<0.001$ ), vascular invasion 

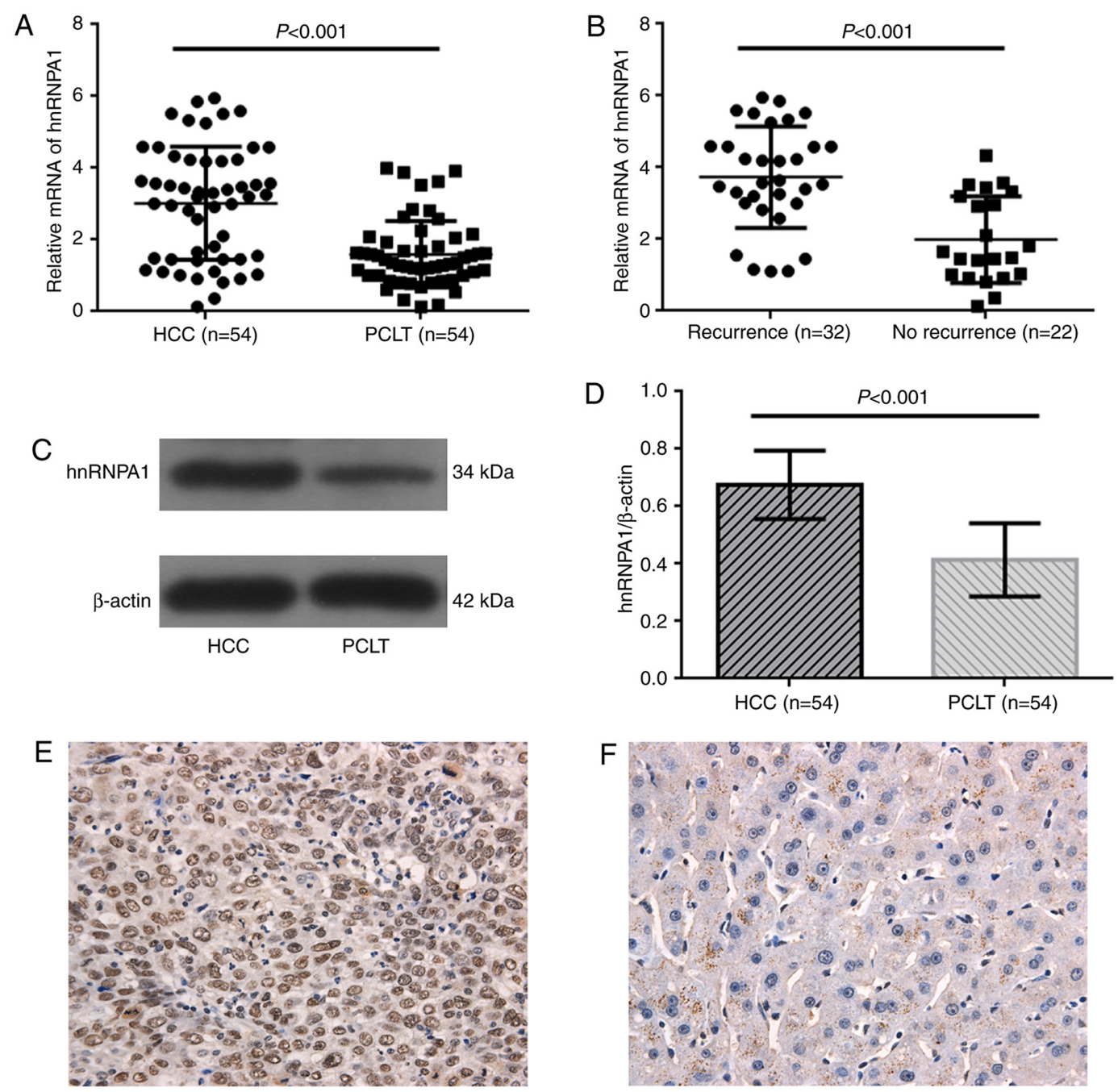

Figure 1. hnRNPA1 is upregulated in HCC tissues. (A) Relative hnRNPA1 RNA levels between tumor tissues and PCLT (P<0.001). (B) Relative hnRNPA1 RNA levels between patients with recurrence and no recurrence $(\mathrm{P}<0.001)$. (C) Confirmation of hnRNPA1/ $\beta$-actin protein expression in a representative case of HCC according to western blotting analysis. (D) The hnRNPA1/ $\beta$-actin level between tumor tissues and corresponding PCLT samples from patients with recurrence and no recurrence $(\mathrm{P}<0.001)$. (E) Representative image of high expression of hnRNPA1 in tumor tissue from a patient with HCC (x400 magnification). (F) Representative image of low expression of hnRNPA1 in tumor tissue from a patient with HCC (x400 magnification). hnRNPA1, heterogeneous nuclear ribonucleoprotein A1; HCC, hepatocellular carcinoma; PCLT, peri-cancerous liver tissues.

(HR, 7.542; 95\% CI, 4.77-11.908; $\mathrm{P}<0.001)$, Edmonson grade (HR, 0.296; 95\% CI, 0.181-0.485; P<0.001), pre-operative blood AFP (HR, 2.122; 95\% CI, 1.594-2.827; P<0.001), NLR (HR, 2.609; 95\% CI, 1.775-3.835; P<0.001), PLR (HR, 2.208; 95\% CI, 1.658-2.941; $\mathrm{P}<0.001)$ and hnRNPA1 expression (HR, 3.184; 95\% CI, 2.244-4.518; $\mathrm{P}<0.001)$ were associated with OS (Table IV). Multivariate analysis confirmed that vascular invasion (HR, 6.118; 95\% CI, 3.729-10.040; $\mathrm{P}<0.001$ ), pre-operative blood AFP (HR, 1.564; 95\% CI, 1.151-2.126; P=0.004), NLR (HR, 1.758; 95\% CI, 1.161-2.661; P=0.008) and hnRNPA1 expression (HR, 0.629; 95\% CI, 0.454-0.871; P=0.005) were independent prognostic factors of OS (Table IV). High hnRNPA1 expression was significantly associated with increased risk of mortality (Table IV).

Association between OS and hIRNPA1, NLR and PLR in patients with HCC during the observation period following curative resection. By the final follow-up, $48.1 \%(205 / 426)$ of the patients had suffered from recurrence and $44.6 \%$ (190/426) had succumbed in cohort 2. The 1-, 3- and 5-year OS rates in the whole cohort were 86.2, 66.7 and 58.9, respectively, and the 1-, 3- and 5-year recurrence rates were 29.6, 45.8 and $47.7 \%$, respectively. Furthermore, it was revealed that patients with HCC with high hnRNPA1 expression had the poorest prognosis at 1, 3 and 5 years, with higher recurrence rates than in patients with low hnRNPA1 expression (51.4 vs. 20.4, 71.4 vs. 54.5 and 90.7 vs. $76.1 \%$, respectively; $\mathrm{P}<0.001$; Fig. $2 \mathrm{~A}$ ). Similarly, the 1-, 3- and 5-year overall survival rates of the patients with low expression of hnRNPA1 were significantly higher than the survival rates of the hnRNPA1 high group (94.6 vs. 80.7, 62.2 vs. 46.7 and 30.5 vs. $15.4 \%$, respectively; P<0.001; Fig. 2 B).

It was also revealed that patients with $\mathrm{HCC}$ with pre-operative NLR $>5$ had the poorest prognosis at 1,3 and 5 years, with higher cumulative recurrence rates than the patients with pre-operative NLR $\leq 5$ (81.4 vs. 96.8, 48.5 vs. 62.4 and 19.9 vs. $24.8 \%$, respectively; $\mathrm{P}<0.001$; Fig. $2 \mathrm{C}$ ). Similarly, the 1-, 3- and 5-year survival rates of the patients with pre-operative PLR >91 were significantly lower than the survival rates of patients with pre-operative PLR $\leq 91$ (73.8 vs. $92.5,38.6$ vs. 60.1 and 13.1 vs. $25.6 \%$, respectively; 
Table II. Association between hnRNPA1 expression and clinicopathological characteristics in $\mathrm{HCC}(\mathrm{n}=426$; cohort 2$)$.

hnRNPA1

expression level

Characteristics n Low, $\mathrm{n}$ High, $\mathrm{n}$ P-value

\begin{tabular}{|c|c|c|c|c|}
\hline \multicolumn{5}{|l|}{ Age, years } \\
\hline$\leq 55$ & 174 & 92 & 160 & 0.170 \\
\hline$>55$ & 252 & 75 & 99 & \\
\hline \multicolumn{5}{|l|}{ Sex } \\
\hline Male & 378 & 149 & 229 & 0.867 \\
\hline Female & 48 & 18 & 30 & \\
\hline \multicolumn{5}{|c|}{ Tumor location } \\
\hline Left & 101 & 41 & 60 & 0.743 \\
\hline Right & 325 & 126 & 199 & \\
\hline \multicolumn{5}{|l|}{ TNM stage } \\
\hline I/II & 292 & 125 & 167 & 0.024 \\
\hline IIIa & 134 & 42 & 92 & \\
\hline \multicolumn{5}{|c|}{ Tumor size, cm } \\
\hline$\leq 5$ & 219 & 97 & 122 & 0.027 \\
\hline$>5$ & 207 & 70 & 137 & \\
\hline \multicolumn{5}{|c|}{ Vascular invasion } \\
\hline Yes & 287 & 94 & 193 & $<0.001$ \\
\hline No & 139 & 73 & 66 & \\
\hline \multicolumn{5}{|c|}{ Edmonson grade } \\
\hline I & 343 & 114 & 229 & $<0.001$ \\
\hline II-IV & 83 & 53 & 30 & \\
\hline \multicolumn{5}{|l|}{$\mathrm{AFP}, \mu \mathrm{g} / 1$} \\
\hline$\leq 400$ & 267 & 126 & 141 & $<0.001$ \\
\hline$>400$ & 159 & 41 & 118 & \\
\hline \multicolumn{5}{|l|}{$\mathrm{HBsAg}$} \\
\hline Negative & 275 & 108 & 167 & 0.968 \\
\hline Positive & 151 & 59 & 92 & \\
\hline \multicolumn{5}{|c|}{ HBV DNA load, IU/ml } \\
\hline$\leq 104$ & 224 & 86 & 138 & 0.719 \\
\hline$>104$ & 202 & 81 & 121 & \\
\hline \multicolumn{5}{|l|}{ NLR } \\
\hline$\leq 5.0$ & 125 & 62 & 63 & 0.005 \\
\hline$>5.0$ & 301 & 105 & 196 & \\
\hline \multicolumn{5}{|l|}{ PLR } \\
\hline$\leq 91$ & 281 & 125 & 156 & 0.002 \\
\hline$>91$ & 145 & 42 & 103 & \\
\hline \multicolumn{5}{|l|}{ Survival } \\
\hline Yes & 236 & 127 & 109 & $<0.001$ \\
\hline No & 190 & 40 & 150 & \\
\hline \multicolumn{5}{|l|}{ Recurrence } \\
\hline Yes & 205 & 57 & 148 & $<0.001$ \\
\hline No & 221 & 110 & 111 & \\
\hline
\end{tabular}

TNM, Tumor-Node-Metastasis; AFP, $\alpha$-fetoprotein; NLR, neutrophil to lymphocyte ratio; PLR, platelet to lymphocyte ratio; HBV, hepatitis $B$ virus; HBsAg, HBV surface antigen; hnRNPA1, heterogeneous nuclear ribonucleoprotein A1.
$\mathrm{P}<0.001$; Fig. 2D). The expression of hnRNPA1 was also positively correlated with NLR (Spearman's correlation; $r=0.122$, $\mathrm{P}=0.012$ ) and PLR (Spearman's correlation; $\mathrm{r}=0.140, \mathrm{P}=0.004$ ).

\section{Discussion}

HCC is a highly malignant type of cancer with poor survival rates. There are few HCC-specific biomarkers that are used in clinical practice, but the present study has revealed several potential prognostic indicators. In an attempt to identify a prognostic marker for HCC, hnRNPA1 expression was studied in tissue samples from patients with RHCC. hnRNPA1 is a nucleic acid binding protein that is distributed in various human tissues and organs. The selective splicing of hnRNPA1 is regulated throughout the human transcriptome and uses several mechanisms in conjunction with several splicing factors (24). It has been reported that knocking down hnRNPA1 and its downstream target pyruvate kinase muscle isozyme M2 (PKM2) can inhibit the growth of cancer cells (25). PKM2 promotes glucose metabolism and cancer cell growth through the let-7a/c-Myc/hnRNPA1 feedback loop mechanism (26). In addition, hnRNPA1 may prevent tumor cells from entering senescence by binding to telomeric DNA sequences (27).

The present study investigated the expression of hnRNPA1 in two cohorts of clinical HCC samples. In the 54 tumors samples from cohort 1, hnRNPA1 mRNA was highly expressed in the HCC tissues compared with that in the corresponding PCLTs. Samples from patients with recurrent HCC exhibited higher levels of hnRNPA1 than those without recurrence. In another independent cohort (cohort 2), similar results were obtained at the protein level. Another finding from the present study was the correlation between hnRNPA1 expression and a poor prognosis for HCC patients; overexpression of hnRNPA1 in HCC patients predicted lower OS rates and higher recurrence rates. Elevated hnRNPA1 expression was significantly associated with pre-operative blood AFP levels, TNM stage, tumor size, vascular invasion, Edmonson grade, NLR, PLR, survival and recurrence, but not with patient clinicopathological characteristics. These findings indicate an important role of hnRNPA1 gene in the recurrence of liver cancer. It is known that hnRNPA1 can activate the epidermal growth factor receptor/mitogen-activated protein kinase (MAPK)/extracellular signal-regulated kinase (ERK) pathway in HCC cells to regulate the splicing of the insulin receptor transcript, as well as the Ras/MAPK/ERK pathway to regulate functional A-Raf gene splicing to stimulate tumor proliferation. Another study revealed that hnRNPA1/A2 overexpression can induce malignant transformation of immortalized hepatocytes and inhibit the apoptosis of HCC cells. If the inhibition of hnRNPA1/A2 expression can reduce in vitro growth and in vivo tumorigenesis of $\mathrm{HCC}$ cells, it is highly likely that it is required for HCC growth and development (28).

The present study also analyzed the effect of hnRNPA1 expression on tumor recurrence and metastasis in HCC patients, and revealed that the recurrence rate in the hnRNPA1-positive group was significantly higher, and that the survival rate was significantly lower compared with that in the hnRNPA1-negative group $(\mathrm{P}<0.001)$, indicating that hnRNPA1 overexpression may present a potential risk 

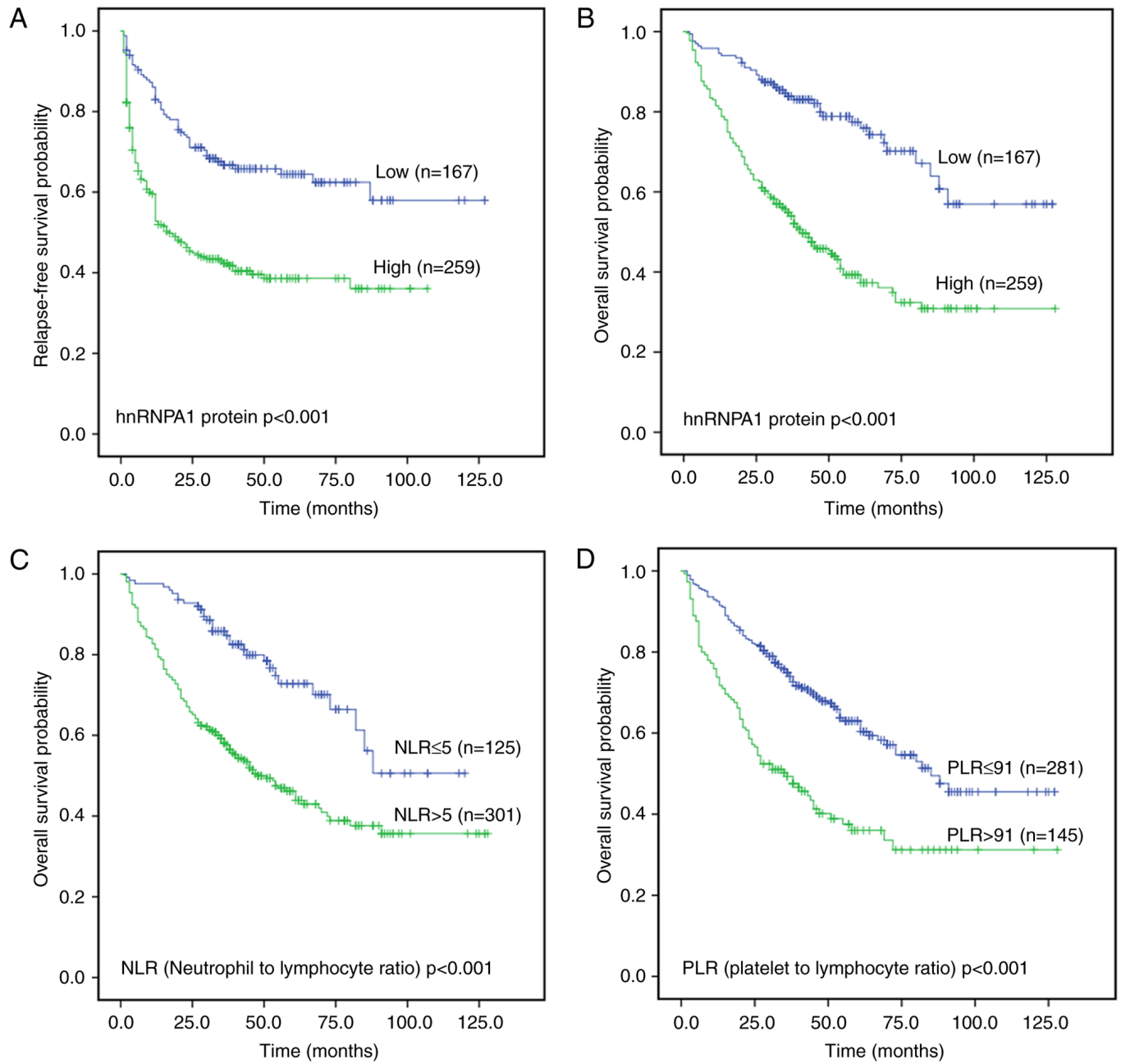

Figure 2. Kaplan-Meier curves of survival differences among HCC patients. The HCC patients with high hnRNPA1 expression had poorer prognoses, as measured by (A) relapse-free survival and (B) overall survival ( $\mathrm{P}<0.001$ ). (C) Comparison of overall survival between NLR $\leq 5$ group and NLR $>5$ group in HCC patients $(\mathrm{P}<0.001)$. (D) Comparison of overall survival between PLR $\leq 91$ group and PLR $>91$ group in HCC patients $(\mathrm{P}<0.001)$. HCC, hepatocellular carcinoma; hnRNPA1, heterogeneous nuclear ribonucleoprotein A1; NLR, neutrophil to lymphocyte ratio; PLR, platelet to lymphocyte ratio.

of recurrence and metastasis. Multivariate analyses validated hnRNPA1 as a significant independent predictor for RFS (HR, 0.685; 95\% CI, 0.506-0.928; $\mathrm{P}=0.015)$ and $\mathrm{OS}$ (HR, 0.629; 95\% CI, 0.454-0.871; $\mathrm{P}=0.005$ ). These observations were similar to those of previous reports on HCC (14) and other malignancies (10-13). Another study (29) reported a major role of hnRNPA1 in the majority of tumors, along with its association with metastasis in different cancer types. Furthermore, overexpression of hnRNPA1 promoted tumor invasion through regulating CD44v6, which indicated that hnRNPA1 may act as a marker for tumor aggressiveness and as a prognostic predictor in HCC (14).

Currently, the association between hnRNPA1 and the clinicopathological characteristics of $\mathrm{HCC}$ remains controversial. AFP is a well-known HCC biomarker used for monitoring treatment, and patients with elevated serum AFP frequently have a worse prognosis for OS (30). The risk of mortality increased significantly in patients with higher pre-operative serum AFP in the present study. Multivariate analyses validated that a pre-operative blood AFP level $>400 \mu \mathrm{g} / 1$ was an independent prognostic factor for RFS (HR, 1.350; 95\% CI, 1.006-1.811; $\mathrm{P}=0.045)$ and OS (HR,
1.564; 95\% CI, 1.151-2.126; $\mathrm{P}=0.004)$. The results of the present study also revealed a significant association between elevated hnRNPA1 expression and elevated pre-operative blood AFP. Therefore, the combination of hnRNPA1 expression and serum AFP presents a good biomarker for this specific population.

Elevated pre-operative AFP, tumor diameter $>5 \mathrm{~cm}$ and vascular invasion are three widely recognized factors affecting the prognosis of liver cancer patients, and have been incorporated into the guidelines for liver cancer diagnosis and treatment (31). Inflammation serves an important role in tumor formation, progression and invasion (32); the inflammatory cytokines released by immune cells can trigger oxidative damage and malignant transformation, and aggravate the inflammatory state, which enhances tumor growth, tissue invasion and metastasis $(15,33)$. Inflammatory factors released by tumor cells also stimulate tumorigenesis, tumor angiogenesis, invasion and metastasis (34). Neutrophils, an important mediator of inflammatory reactions, can further promote tumor growth and invasion by remodeling the extracellular matrix, which significantly inhibits lymphocyte-mediated tumor cell death. Platelet-reactive protein 
Table III. Association between recurrence/survival and patient characteristics.

\begin{tabular}{|c|c|c|c|c|c|c|}
\hline \multirow[b]{2}{*}{ Characteristics } & \multicolumn{3}{|c|}{ Survival } & \multicolumn{3}{|c|}{ Recurrence } \\
\hline & Dead, n & Alive, $\mathrm{n}$ & P-value & Yes, $\mathrm{n}$ & No, $n$ & P-value \\
\hline \multicolumn{7}{|l|}{ Age, years } \\
\hline$\leq 55$ & 115 & 137 & 0.613 & 134 & 118 & 0.006 \\
\hline$>55$ & 75 & 99 & & 71 & 103 & \\
\hline \multicolumn{7}{|l|}{ Sex } \\
\hline Male & 174 & 204 & 0.135 & 185 & 193 & 0.292 \\
\hline Female & 16 & 32 & & 20 & 28 & \\
\hline \multicolumn{7}{|l|}{ Tumor location } \\
\hline Left & 34 & 67 & 0.053 & 46 & 55 & 0.483 \\
\hline Right & 156 & 169 & & 159 & 166 & \\
\hline \multicolumn{7}{|l|}{ TNM stage } \\
\hline $\mathrm{I} / \mathrm{II}$ & 104 & 188 & $<0.001$ & 125 & 167 & $<0.001$ \\
\hline IIIa & 86 & 48 & & 80 & 54 & \\
\hline \multicolumn{7}{|l|}{ Tumor size, $\mathrm{cm}$} \\
\hline$\leq 5$ & 70 & 149 & $<0.001$ & 88 & 131 & $<0.001$ \\
\hline$>5$ & 120 & 87 & & 117 & 90 & \\
\hline \multicolumn{7}{|c|}{ Vascular invasion } \\
\hline Yes & 166 & 121 & $<0.001$ & 181 & 106 & $<0.001$ \\
\hline No & 24 & 115 & & 24 & 115 & \\
\hline \multicolumn{7}{|l|}{ Edmonson grade } \\
\hline $\mathrm{I}$ & 172 & 171 & $<0.001$ & 175 & 168 & 0.003 \\
\hline II-IV & 18 & 65 & & 30 & 53 & \\
\hline \multicolumn{7}{|l|}{$\mathrm{AFP}, \mu \mathrm{g} / 1$} \\
\hline$\leq 400$ & 99 & 168 & $<0.001$ & 115 & 152 & $<0.001$ \\
\hline$>400$ & 91 & 68 & & 90 & 69 & \\
\hline \multicolumn{7}{|l|}{ HBsAg } \\
\hline Negative & 126 & 149 & 0.495 & 136 & 139 & 0.458 \\
\hline Positive & 64 & 87 & & 69 & 82 & \\
\hline \multicolumn{7}{|c|}{ HBV DNA load, IU/ml } \\
\hline$\leq 104$ & 90 & 134 & 0.053 & 109 & 105 & 0.243 \\
\hline$>104$ & 100 & 102 & & 96 & 116 & \\
\hline \multicolumn{7}{|l|}{ NLR } \\
\hline$\leq 5.0$ & 31 & 94 & $<0.001$ & 55 & 70 & 0.031 \\
\hline$>5.0$ & 159 & 142 & & 150 & 151 & \\
\hline \multicolumn{7}{|l|}{ PLR } \\
\hline$\leq 91$ & 103 & 178 & $<0.001$ & 134 & 147 & 0.095 \\
\hline$>91$ & 87 & 58 & & 71 & 74 & \\
\hline \multicolumn{7}{|l|}{ hnRNPA1 } \\
\hline Low & 40 & 127 & $<0.001$ & 57 & 110 & $<0.001$ \\
\hline High & 109 & 150 & & 148 & 111 & \\
\hline
\end{tabular}

OS and RFS were analyzed by Kaplan-Meier survival and log-rank analyses for all characteristics. $\mathrm{P}<0.05$ was considered to indicate a statistically significant difference. OS, overall survival; RFS, relapse-free survival; TNM, Tumor-Node-Metastasis; AFP, $\alpha$-fetoprotein; NLR, neutrophil to lymphocyte ratio; PLR, platelet to lymphocyte ratio; HBV, hepatitis B virus; HBsAg, HBV surface antigen; hnRNPA1, heterogeneous nuclear ribonucleoprotein A1.

and other factors released by the platelets also encourage the proliferation of malignant tumor cells (35). Therefore, when the NLR and/or PLR increases, the immune system is weakened and cannot clear the tumor cells. Studies have shown that pre-operative elevated NLR and PLR are associated with poor prognosis in patients with liver cancer $(36,37)$. 
Table IV. Univariate and multivariate Cox regression analysis of RFS and OS.

\begin{tabular}{|c|c|c|c|c|}
\hline \multirow[b]{2}{*}{ Variables } & \multicolumn{2}{|l|}{ RFS } & \multicolumn{2}{|l|}{ OS } \\
\hline & $\mathrm{HR}(95 \% \mathrm{CI})$ & P-value & $\mathrm{HR}(95 \% \mathrm{CI})$ & P-value \\
\hline \multicolumn{5}{|l|}{ Univariate analysis ${ }^{\mathrm{a}}$} \\
\hline Age, years ( $\leq 55$ vs. $>55)$ & $1.476(1.107-1.968)$ & 0.008 & $0.928(0.694-1.242)$ & 0.615 \\
\hline Sex (male vs female) & $0.785(0.495-1.245)$ & 0.303 & $0.680(0.407-1.134)$ & 0.140 \\
\hline Tumor location (left vs. right) & $1.122(0.808-1.558)$ & 0.491 & $1.437(0.991-2.084)$ & 0.056 \\
\hline TNM stage (I/II vs. IIIa) & $1.978(1.491-2.624)$ & $<0.001$ & $2.357(1.770-3.140)$ & $<0.001$ \\
\hline Tumor size, $\mathrm{cm}(\leq 5$ vs. $>5)$ & $1.898(1.438-2.506)$ & $<0.001$ & $2.270(1.690-3.050)$ & $<0.001$ \\
\hline Vascular invasion (yes vs. no) & $6.850(4.352-10.780)$ & $<0.001$ & $7.542(4.77-11.908)$ & $<0.001$ \\
\hline Edmonson grade (I vs. II-IV) & $0.563(0.381-0.831)$ & 0.004 & $0.296(0.181-0.485)$ & $<0.001$ \\
\hline AFP, $\mu \mathrm{g} / 1$ ( $\leq 400$ vs. $>400)$ & $1.742(1.320-2.300)$ & $<0.001$ & $2.122(1.594-2.827)$ & $<0.001$ \\
\hline HBsAg (negative vs positive) & $0.875(0.654-1.170)$ & 0.367 & $0.853(0.631-1.154)$ & 0.303 \\
\hline HBV DNA load, IU/ml ( $\leq 104$ vs. $>104)$ & $0.946(0.719-1.244)$ & 0.699 & $1.231(0.926-1.637)$ & 0.153 \\
\hline NLR ( $\leq 5.0$ vs. $>5.0)$ & $1.394(1.023-1.899)$ & 0.035 & $2.609(1.775-3.835)$ & $<0.001$ \\
\hline $\operatorname{PLR}(\leq 91$ vs. $>91)$ & $1.271(0.952-1.695)$ & 0.103 & $2.208(1.658-2.941)$ & $<0.001$ \\
\hline hnRNPA1 (low vs high) & $2.295(1.688-3.120)$ & $<0.001$ & $3.184(2.244-4.518)$ & $<0.001$ \\
\hline \multicolumn{5}{|l|}{ Multivariate analysis $^{\mathrm{a}}$} \\
\hline Age, years ( $\leq 55$ vs. $>55)$ & $0.654(0.488-0.877)$ & 0.005 & & \\
\hline TNM stage (I/II vs. IIIa) & $1.411(0.950-2.094)$ & 0.088 & $1.218(0.815-1.819)$ & 0.337 \\
\hline Tumor size, $\mathrm{cm}(\leq 5$ vs. $>5)$ & $1.003(0.681-1.478)$ & 0.988 & $1.161(0.763-1.766)$ & 0.487 \\
\hline Vascular invasion (yes vs. no) & $6.125(3.835-9.870)$ & $<0.001$ & $6.118(3.729-10.040)$ & $<0.001$ \\
\hline Edmonson grade (I vs. II-IV) & $1.047(0.688-1.592)$ & 0.831 & $0.643(0.380-1.086)$ & 0.099 \\
\hline AFP, $\mu \mathrm{g} / 1$ ( $\leq 400$ vs. $>400)$ & $1.350(1.006-1.811)$ & 0.045 & $1.564(1.151-2.126)$ & 0.004 \\
\hline NLR ( $\leq 5.0$ vs. $>5.0)$ & $1.020(0.734-1.417)$ & 0.907 & $1.758(1.161-2.661)$ & 0.008 \\
\hline PLR ( $\leq 91$ vs. $>91)$ & & & $1.343(0.978-1.846)$ & 0.069 \\
\hline hnRNPA1 (low vs. high) & $0.685(0.506-0.928)$ & 0.015 & $0.629(0.454-0.871)$ & 0.005 \\
\hline
\end{tabular}

${ }^{a}$ Cox proportional hazards regression. $\mathrm{P}<0.05$ was considered to indicate a statistically significant difference. RFS, relapse-free survival; OS, overall survival; AFP, $\alpha$-fetoprotein; NLR, neutrophil to lymphocyte ratio; PLR, platelet to lymphocyte ratio; TNM, Tumor-Node-Metastasis; HR, hazard ratio; CI, confidential interval; HBV, hepatitis B virus; HBsAg, HBV surface antigen; hnRNPA1, heterogeneous nuclear ribonucleoprotein A1.

As biological indicators of systemic inflammation, NLR and PLR reflect the host immunity level. In the present study, pre-operative PLR level was not an independent risk factor for long-term survival, but patients with PLR >91 had significantly lower long-term OS compared with patients with PLR $\leq 91$. This is in agreement with a previous study, which associated pre-operative PLR $\geq 125$ with higher tumor staging and aggressive tumor behavior, and showed it to be an independent prognostic factor for liver cancer recurrence following liver transplantation (38). Nevertheless, the heterogeneity among the selected subjects and PLR truncation methods may explain the differences between the aforementioned studies regarding the use of PLR as an independent risk factor for liver cancer patients. The mortality risk increased significantly in patients with higher pre-operative NLR, and multivariate analysis confirmed that higher pre-operative NLR had an unfavorable impact on OS (HR, 1.758; 95\% CI, 1.161-2.661; $\mathrm{P}=0.008)$. hnRNPA1 expression level was correlated with NLR $(r=0.122, P=0.012)$ and PLR $(r=0.140$, $\mathrm{P}=0.004)$. Taken together, the results of the present study point towards an association between hnRNPA1 expression and other biological markers of systemic inflammation, although the specific mechanism remains unclear.

Potential limitations of the present study include its retrospective nature, single-center sampling and the small sample size (480 patients in two independent cohorts). Therefore, to further validate these findings, a large-scale prospective study should be performed.

In conclusion, elevated hnRNPA1 may be an independent prognostic factor of increased mortality and recurrence risk. Clinical detection of hnRNPA1 levels could therefore be beneficial for the treatment and prognostic evaluation of HCC patients. In the present study, hnRNPA1 protein expression in HCC tissue was associated with AFP, NLR and PLR, and the combination of high hnRNPA1 and pre-operative serum AFP levels was a good biomarker for this specific population. There is also a possibility of a correlation between hnRNPA1 expression and biological markers of systemic inflammation. Future studies should focus on cytological and molecular investigations that may aid further understanding of the mechanism of hnRNPA1-mediated tumorigenesis/progression and unearth novel targets for the treatment of HCC. 


\section{Acknowledgements}

Not applicable.

\section{Funding}

This study was supported by The Key Project of Natural Science Foundation of Fujian Province (grant nos. 2015J01406, 2015 y0026 and 2016J01585), The Army's Logistics Medical Research Major Projects Fund Grants (grant nos. CNJ15J002 and 12ZX22) and Startup Fund for Scientific Research, Fujian Medical University (grant no. 2017XQ2048).

\section{Availability of data and materials}

All data generated or analyzed during this study are included in this published article.

\section{Authors' contributions}

KZ and YJ designed the research. RK, LL, JL and XZ conducted the experiments. RK, LL and FY analyzed the data. The manuscript was drafted by RK and LL. All authors read and approved the final manuscript.

\section{Ethics approval and consent to participate}

The present study was approved by the Human Research Ethics Committee of Fuzhou General Hospital (Fuzhou, China) and written informed consent was obtained from all participants.

\section{Patient consent for publication}

All participants provided written informed consent for publication of the data and any associated images.

\section{Competing interests}

The authors declare that they have no competing interests.

\section{References}

1. Yan X and Qiu Y: Impact of current staging systems on treatment strategy for HBV-related hepatocellular carcinoma. Cancer Lett 379: 220-224, 2016.

2. Chen W, Zheng R, Zhang S, Zhao P, Zeng H and Zou X: Report of cancer incidence and mortality in China, 2010. Ann Transl Med 2: 61, 2014.

3. Olsen SK, Brown RS and Siegel AB: Hepatocellular carcinoma: Review of current treatment with a focus on targeted molecular therapies. Therap Adv Gastroenterol 3: 55-66, 2010.

4. Wang CH, Wey KC, Mo LR, Chang KK, Lin RC and Kuo JJ Current trends and recent advances in diagnosis, therapy, and prevention of hepatocellular carcinoma. Asian Pac J Cancer Prev 16: 3595-3604, 2015.

5. Jonas S, Al-Abadi H, Benckert C, Thelen A,Hippler-Benscheid M, Saribeyoglu K, Radtke B, Pratschke J and Neuhaus P: Prognostic significance of the DNA-index in liver transplantation for hepatocellular carcinoma in cirrhosis. Ann Surg 250: 1008-1013, 2009.

6. Kim E, Goren A and Ast G: Insights into the connection between cancer and alternative splicing. Trends Genet 24: 7-10, 2008.

7. Papaemmanuil E, Cazzola M, Boultwood J, Malcovati L, Vyas P, Bowen D, Pellagatti A, Wainscoat JS, Hellstrom-Lindberg E, Gambacorti-Passerini C, et al: Somatic SF3B1 mutation in myelodysplasia with ring sideroblasts. N Engl J Med 365: 1384-1395, 2011.
8. Han SP, Tang YH and Smith R: Functional diversity of the hnRNPs: Past, present and perspectives. Biochem J 430: 379-392, 2010.

9. LaBranche H, Dupuis S, Ben-David Y, Bani MR, Wellinger RJ and Chabot B: Telomere elongation by hnRNP A1 and a derivative that interacts with telomeric repeats and telomerase. Nat Genet 19: 199-202, 1998.

10. Cammas A, Lacroix-Triki M, Pierredon S, Le Bras M, Iacovoni JS, Teulade-Fichou MP, Favre G, Roché H, Filleron T, Millevoi S and Vagner S: hnRNP A1-mediated translational regulation of the $\mathrm{G}$ quadruplex-containing $\mathrm{RON}$ receptor tyrosine kinase mRNA linked to tumor progression. Oncotarget 7: 16793-16805, 2016.

11. David CJ, Chen M, Assanah M, Canoll P and Manley JL: HnRNP proteins controlled by c-Myc deregulate pyruvate kinase mRNA splicing in cancer. Nature 463: 364-368, 2010.

12. Ma YL, Peng JY, Zhang P, Huang L, Liu WJ, Shen TY, Chen HQ, Zhou YK, Zhang M, Chu ZX and Qin HL: Heterogeneous nuclear ribonucleoprotein A1 is identified as a potential biomarker for colorectal cancer based on differential proteomics technology. J Proteome Res 8: 4525-4535, 2009.

13. Golan-Gerstl R, Cohen M, Shilo A, Suh SS, Bakàcs A, Coppola L and Karni R: Splicing factor hnRNP A2/B1 regulates tumor suppressor gene splicing and is an oncogenic driver in glioblastoma. Cancer Res 71: 4464-4472, 2011.

14. Zhou ZJ, Dai Z, Zhou SL, Fu XT, Zhao YM, Shi YH, Zhou J and Fan J: Overexpression of HnRNP A1 promotes tumor invasion through regulating $\mathrm{CD} 44 \mathrm{v} 6$ and indicates poor prognosis for hepatocellular carcinoma. Int J Cancer 132: 1080-1089, 2013.

15. Tazzyman S, Barry ST, Ashton S, Wood P, Blakey D, Lewis CE and Murdoch C: Inhibition of neutrophil infiltration into A549 lung tumors in vitro and in vivo using a CXCR2-specific antagonist is associated with reduced tumor growth. Int J Cancer 129: 847-858, 2011.

16. Suzuki K, Kachala SS, Kadota K, Shen R, Mo Q, Beer DG, Rusch VW, Travis WD and Adusumilli PS: Prognostic immune markers in non-small cell lung cancer. Clin Cancer Res 17: 5247-5256, 2011.

17. Wei K, Wang $M$, Zhang $W, M u ~ H$ and Song TQ: Neutrophil-lymphocyte ratio as a predictor of outcomes for patients with hepatocellular carcinoma undergoing TAE combined with Sorafenib. Med Oncol 31: 969, 2014.

18. Cao Y, Ke R, Wang S, Zhu X, Chen J, Huang C, Jiang Y and Lv L: DNA topoisomerase IIalpha and Ki67 are prognostic factors in patients with hepatocellular carcinoma. Oncol Lett 13: 4109-4116, 2017.

19. Kee KM, Wang JH, Lee CM, Chen CL, Changchien CS, Hu TH, Cheng YF, Hsu HC, Wang CC, Chen TY, et al: Validation of clinical AJCC/UICC TNM staging system for hepatocellular carcinoma: analysis of 5,613 cases from a medical center in southern Taiwan. Int J Cancer 120: 2650-2655, 2007.

20. Livak KJ and Schmittgen TD: Analysis of relative gene expression data using real-time quantitative PCR and the 2(-Delta Delta C(T)) method. Methods 25: 402-408, 2001.

21. Wu X, Liu H, Liu J, Haley KN, Treadway JA, Larson JP, Ge N, Peale F and Bruchez MP: Immunofluorescent labeling of cancer marker Her2 and other cellular targets with semiconductor quantum dots. Nat Biotechnol 21: 41-46, 2003.

22. Kinoshita A, Onoda H, Imai N, Iwaku A, Oishi M, Fushiya N, Koike K, Nishino $\mathrm{H}$ and Tajiri $\mathrm{H}$ : Comparison of the prognostic value of inflammation-based prognostic scores in patients with hepatocellular carcinoma. Br J Cancer 107: 988-993, 2012.

23. Dong L, Bai K, Cao Y, Huang Q, Lv L and Jiang Y: Prognostic value of pre-operative platelet to lymphocyte ratio in patients with resected primary hepatocellular carcinoma. Clin Lab 62: 2191-2196, 2016.

24. Jean-Philippe J, Paz S and Caputi M: hnRNP A1: The Swiss army knife of gene expression. Int J Mol Sci 14: 18999-19024, 2013.

25. Zhang S, Wei JS, Li SQ, Badgett TC, Song YK, Agarwal S, Coarfa C, Tolman C, Hurd L, Liao H, et al: MYCN controls an alternative RNA splicing program in high-risk metastatic neuroblastoma. Cancer Lett 371: 214-224, 2016.

26. Luan W, Wang Y, Chen X, Shi Y, Wang J, Zhang J, Qian J, Li R, Tao T, Wei W, et al: PKM2 promotes glucose metabolism and cell growth in gliomas through a mechanism involving a let-7a/c-Myc/hnRNPA1 feedback loop. Oncotarget 6: 13006-13018, 2015.

27. Ford LP, Wright WE and Shay JW: A model for heterogeneous nuclear ribonucleoproteins in telomere and telomerase regulation. Oncogene 21: 580-583, 2002. 
28. Chettouh H, Fartoux L, Aoudjehane L, Wendum D, Clapéron A Chrétien Y, Rey C, Scatton O, Soubrane O, Conti F, et al: Mitogenic insulin receptor-A is overexpressed in human hepatocellular carcinoma due to EGFR-mediated dysregulation of RNA splicing factors. Cancer Res 73: 3974-3986, 2013.

29. Liu X, Zhou Y, Lou Y and Zhong H: Knockdown of HNRNPA1 inhibits lung adenocarcinoma cell proliferation through cell cycle arrest at G0/G1 phase. Gene 576: 791-797, 2016.

30. Zhen L, Shijie N and Shuijun Z: Tumor PHD2 expression is correlated with clinical features and prognosis of patients with HCC receiving liver resection. Medicine 93: e179, 2014.

31. Vitale A, Morales RR, Zanus G, Farinati F, Burra P, Angeli P, Frigo AC, Del Poggio P, Rapaccini G, Di Nolfo MA, et al: Barcelona clinic liver cancer staging and transplant survival benefit for patients with hepatocellular carcinoma: A multicentre, cohort study. Lancet Oncol 12: 654-662, 2011.

32. Mantovani A, Allavena P, Sica A and Balkwill F: Cancer-related inflammation. Nature 454: 436-444, 2008.

33. Pichler M,Hutterer GC, Stoeckigt C, Chromecki TF, Stojakovic T, Golbeck S, Eberhard K, Gerger A, Mannweiler S, Pummer K and Zigeuner R: Validation of the pre-treatment neutrophil-lymphocyte ratio as a prognostic factor in a large European cohort of renal cell carcinoma patients. Br J Cancer 108: 901-907, 2013.

34. de Visser KE, Eichten A and Coussens LM: Paradoxical roles of the immune system during cancer development. Nat Rey Cancer 6: 24-37, 2006.
35. Aliustaoglu M, Bilici A, Ustaalioglu BB, Konya V, Gucun M, Seker $M$ and Gumus M: The effect of peripheral blood values on prognosis of patients with locally advanced gastric cancer before treatment. Med Oncol 27: 1060-1065, 2010.

36. Gomez D, Farid S, Malik HZ, Young AL, Toogood GJ, Lodge JP and Prasad KR: Preoperative neutrophil-to-lymphocyte ratio as a prognostic predictor after curative resection for hepatocellular carcinoma. World J Surg 32: 1757-1762, 2008.

37. Song W, Wang K, Zhong FP, Fan YW, Peng L and Zou SB: Clinicopathological and prognostic significance of platelet-to-lymphocyte ratio in patients with hepatocellular carcinoma. Oncotarget 7: 81830-81838, 2016.

38. Qi X, Li J, Deng H, Li H, Su C and Guo X: Neutrophil-tolymphocyte ratio for the prognostic assessment of hepatocellular carcinoma: A systematic review and meta-analysis of observational studies. Oncotarget 7: 45283-45301, 2016.

This work is licensed under a Creative Commons Attribution-NonCommercial-NoDerivatives 4.0 International (CC BY-NC-ND 4.0) License. 